\title{
Não realização de citopatológico de colo uterino entre gestantes no extremo sul do Brasil: prevalência e fatores associados
}

\author{
Non-performance of Pap smears among pregnant women \\ in the Extreme South of Brazil: prevalence and associated factors
}

Rodrigo Jacobi Terlan ${ }^{1}$

Juraci Almeida Cesar ${ }^{2}$
${ }^{1}$ Hospital Universitário Dr. Miguel Riet Correia Júnior, Serviço de Clínica Médica, Universidade Federal do Rio Grande (FURG). Canabarro 301, Centro. 96200-190 Rio Grande RS Brasil. drterlan@yahoo.com.br ${ }^{2}$ Programa de PósGraduação em Saúde Pública, Faculdade de Medicina, FURG. Rio Grande RS Brasil.

\begin{abstract}
Cervical cancer is the third most common cause of cancer among women worldwide, and Pap smears are the best screening strategy for its detection. This study evaluated the prevalence and the associated factors of the non-performance of Pap smears among pregnant women above the age of 25 during prenatal care in the municipality of Rio Grande, Brazil, in 2013. The multivariate analysis was performed by Poisson regression evaluated by prevalence ratio (PR). Of the 1,474 pregnant women included in the study, 21.6\% (95\% CI, 19.5\%-23.7\%) had not been screened. The adjusted analysis evidenced a PR for the non-performance among puerperae with 0-4 years schooling of 2.14 (95\% CI, 1.35-3.38) compared to those with 12 years or more. Reporting previous abortion and alcohol use during pregnancy showed a PR of 1.38 (1.10-1.73) and 1.39 (1.04-1.84) of not doing so compared to the other, respectively. Finally, performing 1-5 prenatal visits evidenced a PR of 1.35 (1.03-1.77) compared to the others. A high proportion of pregnant women non-performing Pap smears and non-compliance with the basic recommendation by the Brazilian Ministry of Health has been found. Health professionals should reinforce the need for this test and active search for pregnant women in the community with the profile described herein.
\end{abstract}

Key words Prenatal care, Papanicolaou test, Risk factors, Screening test
Resumo $O$ câncer do colo do útero é o terceiro tumor mais frequente na população feminina e o teste citopatológico $(C P)$ a principal estratégia para o rastreamento. Este estudo mediu a prevalência e os fatores associados à não realização de $C P$ entre gestantes maiores de 25 anos, durante o pré-natal, no município de Rio Grande, RS, em 2013. A análise multivariável utilizou regressão de Poisson avaliada por meio de razão de prevalências (RP). Dentre as 1474 gestantes incluídas no estudo, 21,6\% (IC95\% 19,5\%-23,7\%) não realizaram $C P$ nos últimos três anos. Na análise ajustada, a RP para não realização entre puérperas com escolaridade entre 0-4 anos foi 2,14 (IC95\%:1,35-3,38) em comparação àquelas com 12 anos ou mais. Ter referido aborto prévio e consumido álcool durante a gestação mostrou $R P=$ 1,38 (1,10-1,73) e 1,39 (1,04-1,84) de não fazê-lo em relação às demais, respectivamente. Realizar 1-5 consultas de pré-natal conferiu $R P=1,35$ $(1,03-1,77)$ em relação às demais. Evidencia-se proporção expressiva de não realização de $C P e$ não cumprimento de recomendação básica do Ministério da Saúde. Os profissionais de saúde deveriam reforçar a necessidade de realização deste exame, bem como busca ativa na comunidade das gestantes com o perfil aqui descrito.

Palavras-chave Pré-natal, Papanicolaou, Fatores de risco, Rastreamento 


\section{Introdução}

O câncer do colo do útero é o terceiro tumor mais frequente na população feminina brasileira, atrás, apenas, do câncer de mama e colorretal. É a quarta causa de morte por câncer entre mulheres no Brasil, com taxa de mortalidade de $4,4 / 100.000^{1}$.

Desde que diagnosticado precocemente, somente o câncer de pele tem maior potencial de prevenção e cura que o de colo de útero. A sua principal estratégia de rastreamento é o teste citopatológico convencional (Papanicolaou). Este teste é recomendado para mulheres com idade entre 25 e 64 anos e com periodicidade de três anos para aquelas com dois resultados negativos consecutivos $^{2}$. O tipo de busca predominante para este tipo de serviço no Brasil é oportunística, ou seja, por outras razões que não o diagnóstico precoce da doença. Por esta razão, metade dos casos é diagnosticado em estádios avançados da doença, quando o prognóstico é pior ${ }^{3}$.

Cerca de 20\% das brasileiras com idade entre 25 e 64 anos nunca realizaram um único exame Papanicolaou ${ }^{1}$. Dentre as que já se submeteram a este teste, proporção expressiva já o fez com periodicidade inferior ao recomendado, enquanto outras estão em atraso ${ }^{4}$. A maioria dos estudos abordando este tema busca avaliar a cobertura ${ }^{4-9}$ e o foco ${ }^{4}$ e identificar aquelas com Papanicolaou em atraso. ${ }^{5}$ As faixas etárias estudadas são bastante diferentes, sobretudo quando se inclui gestantes, o que dificulta comparações e impede a determinação da real magnitude do problema ${ }^{5-12}$.

As gestantes constituem um grupo populacional que pode retratar como as diretrizes para o rastreamento vêm sendo seguidas. No Brasil, $90 \%$ das gestantes realizam pelo menos uma consulta de pré-natal, com cerca de metade delas estando em idade em que a realização deste exame é recomendada ${ }^{10}$. Considerando que este exame deveria ser solicitado já na primeira consulta, era de se esperar uma maior taxa de cobertura e, por conseguinte, menores índices de morbimortalidade. Entre todas as mulheres brasileiras, a taxa de óbito por câncer de colo uterino é 5 por 100 mil, índice considerado elevado, apesar de a cobertura deste exame alcançar 78,7\% delas ${ }^{1,13,14}$.

O aumento na taxa de realização de citopalógico de colo uterino poderia levar a uma drástica redução nos índices de morbimortalidade por esta doença ${ }^{15}$. Logo, incentivar a sua realização durante as consultas de pré-natal poderia contribuir para a redução do número de óbitos por esta causa. A Estratégia Saúde da Família, embo- ra tenha contribuído de forma importante para a redução da morbimortalidade por diversas causas no Brasil, ainda não se mostrou capaz de aumentar de forma significativa a cobertura para este tipo de exame ${ }^{16}$. Observa-se aqui inúmeras oportunidades perdidas de realização de citopatológico de colo uterino.

O presente estudo tem por objetivo medir a prevalência e identificar fatores associados a não realização de exame citopatológico de colo uterino entre gestantes que fizeram pelo menos uma consulta de pré-natal, que possuíam 25 anos ou mais de idade e que tiveram filho no município de Rio Grande, RS, ao longo do ano de 2013.

\section{Metodologia}

Este estudo foi realizado em Rio Grande, município com pouco mais de 200 mil habitantes, localizado no extremo sul do estado do Rio Grande do Sul. Noventa e cinco por cento da sua população reside na área urbana e praticamente todos os partos $(99,5 \%)$ ocorrem nas duas únicas maternidades locais.

Desde 2007, vêm sendo realizados, a cada três anos, inquéritos perinatais com o objetivo de avaliar a qualidade da assistência à gestação e ao parto neste município. O ultimo destes estudos foi conduzido em 2013, de onde se originaram os dados apresentados neste artigo.

Este estudo perinatal incluiu todos os nascimentos ocorridos entre 01/01 a 31/12 de 2013, cujo peso ao nascer tenha sido igual ou superior a 500 gramas e/ou alcançado pelo menos 20 semanas de idade gestacional. Além disso, suas mães deveriam residir em área urbana ou rural do município de Rio Grande.

Em virtude de o interesse deste artigo estar relacionado ao citopatológico de colo uterino durante o pré-natal, e de este exame não ser recomendado de rotina para aquelas com até 24 anos de idade, o denominador deste estudo foi constituído somente por puérperas que realizaram pelo menos uma consulta de pré-natal, que possuíam 25 anos ou mais de idade, que nunca haviam realizado o exame ou o haviam realizado há mais de três anos. As mães eram abordadas uma única vez, ainda no hospital, em até 48 horas após o parto.

O cálculo do tamanho amostral foi estimado a partir dos dados oriundos deste mesmo estudo. Considerando o " $n$ " disponível de 1.474 puérperas, prevalência do desfecho (não realização de Papanicolaou nos últimos três anos) de 21,6\%, 
nível de confiança desejado de 95\%, perdas de $3,0 \%$, foi possível trabalhar com uma margem de erro de 2,2 pontos percentuais ${ }^{17}$.

Em relação ao estudo sobre a identificação de fatores associados, para se trabalhar com erro alfa de 0,05 , erro beta de 0,20 , razão expostos/não expostos de 79/21, prevalência de doença entre os não expostos de $23 \%$ e razão de riscos de 1,4 , o estudo deveria incluir pelo menos 1.331 puérperas. Este valor já se encontra acrescido de 15\% para controle de potenciais fatores de confusão e $3,0 \%$ para perdas. Estes cálculos foram realizados utilizando-se do programa Epi Info 6.04 ${ }^{17}$.

Os entrevistadores foram previamente treinados e um estudo piloto foi realizado. As informações buscadas tratavam de características demográficas (idade e cor da pele da mãe e se vivia com companheiro); nível socioeconômico (renda familiar, escolaridade, se exerceu trabalho remunerado durante a gestação); história reprodutiva (paridade, idade por ocasião do primeiro parto, ocorrência de aborto prévio e planejamento da gravidez); hábitos de vida (uso de tabaco e álcool durante a gestação e prática de atividade física). Considerou-se como tendo realizada atividade física a gestante que, sob orientação do médico, enfermeira e/ou de educador físico, praticou de forma regular exercício físico direcionado, específico para a gravidez. Outros exercícios gerais realizados como atividade escolar, no trabalho ou afazeres domésticos não foram considerados). Por fim, foi investigado o tipo de assistência recebida durante a gestação e o parto, que incluiu o número de consultas de pré-natal realizadas, trimestre de início e local de realização das consultas de pré-natal, e se feitas no setor público ou privado).

Os questionários eram duplamente digitados no programa Epi Data 3.177, a comparação feita no Epi Info 6.04 e a análise de consistência e final no $11.0^{18}$.

As análises bruta e ajustada foram feitas por meio da regressão de Poisson, com ajuste robusto da variância ${ }^{19}$. A medida de desfecho foi expressa pela razão de prevalências (RP), intervalo de confiança de 95\% (IC95\%) e valor p do teste de Wald para heterogeneidade e tendência linear. Para a análise ajustada, elaborou-se modelo hierárquico em três níveis pressupondo que aquelas situados no primeiro nível (mais distal ao desfecho) eram sobredeterminantes em relação as de$\mathrm{mais}^{20}$. No primeiro nível foram incluídas as variáveis demográficas (idade, cor da pele e situação conjugal - se vive ou não com companheiro-) e socioeconômicas (renda familiar, escolaridade materna, e realização de trabalho remunerado no período gestacional); no segundo nível, as variáveis relacionadas à vida reprodutiva (paridade - numero de filhos tidos- ocorrência prévia de aborto - espontânea ou induzido -, planejamento da gravidez desta última gravidez e idade por ocasião do primeiro parto) e comportamentais (tabagismo no período gestacional e prática de atividade física especifica à gravidez); no terceiro nível entraram as variáveis relativas à utilização dos serviços de saúde (número de consultas de pré-natal realizadas, trimestre que iniciou estas consultas e tipo de serviço - público ou privado em que fez as consultas de pré-natal). Este modelo seguiu a outros utilizados quando da avaliação de fatores de risco para o mesmo desfecho, com uma única diferença conservadora, ou seja, todas as variáveis incluídas no modelo foram ajustadas no modelo final, independente do p-valor resultante da sua associação com o desfecho (não realização de citopatológico de colo uterino nesta última gravidez) $)^{5,8-12}$. Isto evita o esvaziamento do modelo final e permite avaliar a ocorrência de eventuais confundidores negativos ${ }^{21}$. As análises foram conduzidas no Stata 11.0 com nível de significância de $95 \%$ para testes bicaudais ${ }^{18,21}$.

Cerca de 7\% das entrevistas foram parcialmente refeitas por telefone ou por visita domiciliar à mãe a fim de avaliar a qualidade dos dados coletados. O índice Kappa de concordância para todas as variáveis testadas variou de 0,63 a 0,89 , o que é considerado pelo menos satisfatório ${ }^{21}$.

Às mães era lido o termo de consentimento, em caso de concordância, duas vias eram assinadas ficando uma delas de posse da participante. $\mathrm{O}$ protocolo de pesquisa foi submetido e aprovado pelo Comitê de Ética em Pesquisa na Área da Saúde (CEPAS) da Universidade Federal do Rio Grande. Além disso, garantiu-se a confidencialidade dos dados, a participação voluntária e a possibilidade de deixar o estudo a qualquer momento, sem necessidade de justificativa.

\section{Resultados}

De acordo com o Sistema de Informações sobre Nascidos Vivos (SINASC) houve 2.769 nascimentos cujas mães residiam no município de Rio Grande. Deste total, foi possível entrevistar 2.687 delas, o que revela taxa de perdas da ordem de $3 \%$. Além destas perdas, foram excluídas 73 por não terem realizado uma única consulta de pré-natal e 1140 (42,4\% do total) por possuírem menos de 25 anos de idade. Restaram, portanto, 
1.474 puérperas que possuíam 25 anos ou mais de idade e que haviam realizado pelo menos uma consulta de pré-natal.

A Tabela 1 mostra que a maioria destas mães era de cor da pele branca $(68,1 \%)$, possuíam idade superior a 30 anos $(57,2 \%)$, viviam com com- panheiro $(89,5 \%)$, tinham renda familiar $\geq$ a 4 salários mínimos $(52,2 \%)$, nove anos ou mais de escolaridade $(72,8 \%)$ e haviam exercido trabalho remunerado durante a gravidez (54,9\%). Pouco mais de um terço delas $(34,2 \%)$ era primípara, mais da metade $(53,6 \%)$ teve o primeiro filho

Tabela 1. Características das gestantes maiores de 25 anos que fizeram pelo menos uma consulta de pré-natal do município de Rio Grande, RS, 2013.

\begin{tabular}{|c|c|c|}
\hline Variáveis & $\%$ & $\mathbf{n}$ \\
\hline \multicolumn{3}{|l|}{ Idade da mãe (anos) } \\
\hline 25 a 29 & 42,8 & 632 \\
\hline 30 a 34 & 35,2 & 519 \\
\hline 35 ou mais & 22,0 & 323 \\
\hline \multicolumn{3}{|l|}{ Cor da pele } \\
\hline Branca & 68,1 & 1005 \\
\hline Parda & 21,8 & 321 \\
\hline Preta/Parda & 10,1 & 148 \\
\hline Situação conjugal/Vivem com marido/companheiro & 89,5 & 1318 \\
\hline \multicolumn{3}{|l|}{ Renda familiar mensal em salários mínimos } \\
\hline Ate 0,9 & 3,1 & 46 \\
\hline 1 a 1,9 & 21,7 & 319 \\
\hline 2 a 3,9 & 23,0 & 340 \\
\hline 4 ou mais & 52,2 & 769 \\
\hline \multicolumn{3}{|l|}{ Escolaridade (em anos) } \\
\hline 0 a 4 & 6,1 & 90 \\
\hline 5 a 8 & 22,1 & 326 \\
\hline 9 a 11 & 48,2 & 710 \\
\hline 12 ou mais & 23,6 & 348 \\
\hline Exerceram trabalho remunerado durante a gravidez & 54,9 & 808 \\
\hline \multicolumn{3}{|l|}{ Número de filhos tidos } \\
\hline 1 & 34,2 & 502 \\
\hline 2 & 33,8 & 498 \\
\hline 3 & 16,0 & 237 \\
\hline 4 ou mais & 16,0 & 237 \\
\hline \multicolumn{3}{|l|}{ Idade em anos no primeiro parto $(\mathrm{n}=1020)$} \\
\hline 13 a 16 & 20,5 & 209 \\
\hline 17 a 19 & 33,1 & 338 \\
\hline 20 a 24 & 29,1 & 297 \\
\hline Mais de 25 & 17,3 & 176 \\
\hline Ocorrência de aborto prévio & 29,4 & 300 \\
\hline \multicolumn{3}{|l|}{ Comportamentais } \\
\hline Tabagismo & 16,3 & 239 \\
\hline Uso de álcool & 9,0 & 132 \\
\hline Praticaram exercício físico (antes ou durante) & 31 & 457 \\
\hline \multicolumn{3}{|l|}{ Número de consultas de pré-natal realizadas } \\
\hline 1 a 5 & 11,8 & 174 \\
\hline 6 ou mais & 88,2 & 1300 \\
\hline Iniciaram as consultas de pré-natal no primeiro trimestre & 82,1 & 1209 \\
\hline \multicolumn{3}{|l|}{ Local de realização do pré-natal } \\
\hline Privado & 57,5 & 847 \\
\hline Público & 42,5 & 627 \\
\hline Prevalência de não realização citopatológico de colo uterino & 21,6 & 319 \\
\hline Total & 100,0 & 1.474 \\
\hline
\end{tabular}


ainda na adolescência, cerca de 30\% referiu ocorrência previa de aborto, 16,3\% disseram-se tabagistas e quase um terço afirmou praticar algum tipo de exercício físico antes ou durante a gestação. Em relação ao pré-natal, pelo menos oito em cada 10 delas realizaram seis ou mais consultas, tendo-as iniciado ainda no primeiro trimestre da gravidez. Por fim, 21,6\% (IC95\%:19,5\%-23,7\%) não realizaram exame citopatológico de colo uterino nos últimos três anos.

A Tabela 2 mostra a prevalência do desfecho por categoria e as análises bruta e ajustada. A prevalência de não realização de citopatológico de colo uterino variou de $13,0 \%$ entre aquelas com 12 anos ou mais de escolaridade a 38,5\% dentre aquelas que iniciaram o pré-natal no terceiro trimestre da gestação. Após ajuste para variáveis do mesmo nível e de níveis anteriores, puérperas com escolaridade entre 0 e 4 anos mostraram que a prevalência de não realização de citopatológico de colo uterino foi 2,4 (IC95\%:1,35-3,38) vezes maior que o valor obtido entre àquelas com 12 anos ou mais de escolaridade; ter referido aborto em algum momento no passado e ingerido be- bida de álcool durante esta gravidez revelaram que a prevalência de não realização de CP foi de $1,38(1,10-1,73)$ e de $1,39(1,04-1,84)$ vezes maior que o valor obtido em relação à mães que não ingeriram bebida alcoólica e que não sofreram qualquer tipo de aborto, respectivamente. Por fim, mães que realizaram entre 1 e 5 consultas de pré-natal mostraram que a prevalência de não realização de CP neste grupo foi $1,35(1,03-1,77)$ vezes o valor obtido em relação ao grupo de referência, ou seja, para aquelas que completaram seis ou mais consulta durante todo o pré-natal.

\section{Discussão}

Duas em cada 10 gestantes, mesmo tendo realizado pelo menos uma consulta de pré-natal, não foram submetidas ao exame citopatológico de colo uterino. E deveriam tê-lo feito. Os principais fatores associados à sua não realização foram baixa escolaridade, ocorrência previa de aborto, consumo de álcool durante a gestação e baixo número de consultas de pré-natal.

Tabela 2. Análises bruta e ajustada para não realização de citopatológico de colo uterino nesta ultima gravidez entre gestantes com 25 anos ou mais de idade que fizeram pelo menos uma consulta de pré-natal em Rio Grande, RS, em 2013. $(\mathrm{n}=1474)$

\begin{tabular}{|c|c|c|c|c|}
\hline \multirow[b]{2}{*}{ Nível } & \multirow[b]{2}{*}{ Variável } & \multirow{2}{*}{$\begin{array}{c}\text { Prevalência de } \\
\text { não realização de } \\
\text { Papanicolaou(\%) }\end{array}$} & \multicolumn{2}{|c|}{ Razão de prevalências (IC95\%) } \\
\hline & & & Bruta & Ajustada \\
\hline \multirow[t]{22}{*}{ I } & Idade da mãe (anos) & & $\mathrm{p}=0,994$ & $\mathrm{p}=0,866$ \\
\hline & 25 a 29 & 21,6 & 1,00 & 1,00 \\
\hline & 30 a 34 & 21,8 & $1,01(0,81-1,26)$ & $1,06(0,85-1,32)$ \\
\hline & 35 ou mais & 21,7 & $1,00(0,78-1,29)$ & $1,03(0,80-1,33)$ \\
\hline & Cor da pele & & $\mathrm{p}=0,046$ & $\mathrm{p}=0,399$ \\
\hline & Branca & 20,2 & 1,00 & 1,00 \\
\hline & Preta/parda & 24,8 & $1,23(1,00-1,49)$ & $1,09(0,88-1,34)$ \\
\hline & Situação conjugal & & $\mathrm{p}=0,584$ & $\mathrm{p}=0,859$ \\
\hline & Vive com companheiro & 23,4 & 1,00 & 1,00 \\
\hline & Vive sem companheiro & 21,5 & $0,92(0,68-1,24)$ & $0,97(0,71-1,34)$ \\
\hline & Renda familiar em tercis & & $\mathrm{p}<0,001$ & $\mathrm{p}=0,229$ \\
\hline & Primeiro & 24,9 & 1,00 & 1,00 \\
\hline & Segundo & 25,7 & $0,68(0,53-0,88)$ & $1,17(0,92-1,50)$ \\
\hline & Terceiro (melhor) & 17,1 & $0,25(0,21-0,29)$ & $0,97(0,72-1,30)$ \\
\hline & Escolaridade materna (anos) & & $\mathrm{p}<0,001$ & $\mathrm{p}=0,020$ \\
\hline & 0 a 4 & 31,1 & $2,40(1,59-3,62)$ & $2,14(1,35-3,38)$ \\
\hline & 5 a 8 & 28,5 & $2,20(1,59-3,04)$ & $1,98(1,37-2,85)$ \\
\hline & 9 a 11 & 21,6 & $1,66(1,22-2,26)$ & $1,55(1,13-2,14)$ \\
\hline & 12 ou mais & 13,0 & 1,00 & 1,00 \\
\hline & Atividade remunerada durante a gravidez & & $\mathrm{p}=0,005$ & $\mathrm{p}=0,268$ \\
\hline & Não & 25,0 & 1,00 & 1,00 \\
\hline & Sim & 18,9 & $0,76(0,62-0,92)$ & $0,89(0,71-1,10)$ \\
\hline
\end{tabular}


Tabela 2. Análises bruta e ajustada para não realização de citopatológico de colo uterino nesta ultima gravidez entre gestantes com 25 anos ou mais de idade que fizeram pelo menos uma consulta de pré-natal em Rio Grande, RS, em 2013. ( $\mathrm{n}=1474)$.

\begin{tabular}{|c|c|c|c|c|}
\hline \multirow[b]{2}{*}{ Nível } & \multirow[b]{2}{*}{ Variável } & \multirow{2}{*}{$\begin{array}{c}\text { Prevalência de } \\
\text { não realização de } \\
\text { Papanicolaou(\%) }\end{array}$} & \multicolumn{2}{|c|}{ Razão de prevalências (IC95\%) } \\
\hline & & & Bruta & Ajustada \\
\hline \multirow[t]{26}{*}{ II } & Numero de filhos tidos & & $\mathrm{p}<0,001$ & $\mathrm{p}=0,227$ \\
\hline & 1 & 15,1 & 1,00 & 1,00 \\
\hline & 2 & 20,5 & $1,35(1,03-1,77)$ & $1,53(0,73-3,19)$ \\
\hline & 3 & 28,2 & $1,87(1,40-2,50)$ & $1,88(0,88-4,01)$ \\
\hline & 4 ou mais & 31,3 & $2,06(1,56-2,73)$ & $1,89(0,87-4,09)$ \\
\hline & Ocorrência previa de aborto & & $\mathrm{p}=0,008$ & $\mathrm{p}=0,005^{\star}$ \\
\hline & Não & 22,2 & 1,00 & 1,00 \\
\hline & Sim & 30,0 & $1,35(1,08-1,68)$ & $1,38(1,10-1,73)$ \\
\hline & Planejamento da gestação atual & & $\mathrm{p}=0,003$ & $\mathrm{p}=0,954$ \\
\hline & Sim & 18,1 & 1,00 & 1,00 \\
\hline & Não & 24,5 & $1,35(1,10-1,65)$ & $0,99(0,78-1,26)$ \\
\hline & Idade do primeiro parto (anos) & & $p=0,012$ & $\mathrm{p}=0,649$ \\
\hline & 13 a 16 & 29,7 & $1,86(1,35-2,78)$ & $1,25(0,81-1,94)$ \\
\hline & 17 a 19 & 27,2 & $1,71(1,17-2,50)$ & $1,30(0,86-1,97)$ \\
\hline & 20 a 24 & 22,9 & $1,44(0,97-2,14)$ & $1,26(0,84-1,89)$ \\
\hline & 25 ou mais & 15,9 & 1,00 & 1,00 \\
\hline & Tabagismo & & $\mathrm{p}<0,001$ & $\mathrm{p}=0,548$ \\
\hline & Não & 19,1 & 1,00 & 1,00 \\
\hline & Ex-tabagista & 24,2 & $1,27(0,96-1,68)$ & $1,10(0,81-1,50)$ \\
\hline & Tabagista & 31,0 & $1,62(1,29-2,04)$ & $1,16(0,88-1,52)$ \\
\hline & Uso de álcool & & $\mathrm{p}<0,001$ & $\mathrm{p}=0,025^{\circ}$ \\
\hline & Não & 20,5 & 1,00 & 1,00 \\
\hline & $\operatorname{Sim}$ & 34,1 & $1,66(1,29-2,16)$ & $1,39(1,04-1,84)$ \\
\hline & Atividade física específica à gestação & & $\mathrm{p}=0,022$ & $\mathrm{p}=0,664$ \\
\hline & Não & 23,5 & 1,00 & 1,00 \\
\hline & Sim & 17,9 & $0,77(0,61-0,96)$ & $0,95(0,74-1,21)$ \\
\hline \multirow[t]{10}{*}{ III } & Número de consultas de pré-natal realizadas & & $\mathrm{p}<0,001$ & $\mathrm{p}=0,028^{*}$ \\
\hline & 1 a 5 & 35,1 & $1,77(1,40-2,22)$ & $1,35(1,03-1,77)$ \\
\hline & 6 ou mais & 19,8 & 1,00 & 1,00 \\
\hline & Trimestre de inicio das consultas de pré-natal & & $\mathrm{p}<0,001$ & $\mathrm{p}=0,275$ \\
\hline & Primeiro & 19,3 & 1,00 & 1,00 \\
\hline & Segundo & 31,9 & $1,66(1,33-2,06)$ & $1,23(0,94-1,60)$ \\
\hline & Terceiro & 38,5 & $1,99(1,21-3,29)$ & $1,27(0,74-2,17)$ \\
\hline & Local de realização do pré-natal & & $\mathrm{p}=0,050$ & $\mathrm{p}=0,07$ \\
\hline & Privado & 19,8 & 1,00 & 1,00 \\
\hline & Público & 24,1 & $1,21(0,99-1,47)$ & $0,81(0,64-1,02)$ \\
\hline
\end{tabular}

\# Teste de Wald para tendência linear; * Teste de Wald para heterogeneidade.

Ao interpretar os resultados aqui apresentados, há que considerar pelo menos três aspectos em relação à realização deste exame: 1) pode haver dificuldade para algumas mulheres diferenciar exame ginecológico do teste Papanicolaou; 2) possibilidade de viés de informação e recordatório, ambos condicionados ao relato por parte da entrevistada e 3) superestimação da sua realização em virtude de ser esta a conduta que delas se espera ${ }^{11}$.
Apesar destas limitações, decidiu-se pela sua realização em virtude de a maioria dos estudos que tratam deste tema utilizarem igual metodologia, o que facilita a comparabilidade e de o autorrelato ser bastante preciso ${ }^{22}$. Além disso, este inquérito incluiu todas as gestantes de um município de porte médio, situação única no Brasil, permitiu estimar a cobertura real e não somente o número de procedimentos realizados, como ocorre nas estatísticas oficiais, alcançou alta taxa 
de respostas e de ter lidado com um grupo específico da população que, mesmo visitando o serviço de saúde de forma sistemática e durante varias vezes, boa parte delas não foi submetida a este exame. Finalmente, não se encontrou no Brasil um único estudo de base populacional que tenha incluído somente gestantes dentro da faixa etária recomendada pelo Ministério da Saúde para realização de $\mathrm{CP}$ de colo uterino.

A prevalência de não realização de Papanicolaou nesta população foi de $21,6 \%$. Há pelo menos dois problemas que dificultam a comparação destes resultados. O primeiro é a pequena quantidade de estudos entre gestantes ${ }^{5,23,24}$, enquanto o segundo é a inclusão de mulheres com faixa etária diferente daquela preconizada pelo Ministério da Saúde, ou seja, não somente a partir dos 25 anos de idade.

Estudo conduzido em 2010 neste mesmo município, também com gestantes, encontrou taxa de não realização de CP de $33 \%{ }^{5}$. Esta diferença cerca de $50 \%$ maior se deve a ao fato de este estudo ter incluído todas as gestantes, independente da idade e, portanto, desconsiderando a recomendação do Ministério. Isto eleva a taxa de não realização em virtude da não obrigatoriedade de realizá-lo antes dos 25 anos. Estudo entre gestantes em 2010, no município de Juiz de Fora (MG), encontrou prevalência de não realização de $26,6 \%$. No entanto, dentre aquelas em atraso, $53,6 \%$ possuíam até 24 anos de idade ${ }^{23}$. Em Pelotas, município vizinho, estudo realizado em 2003, que incluiu mulheres entre 20 e 59 anos, mostrou taxa de não realização de $17,0 \%{ }^{9}$. Neste, além da faixa etária, há diferença de 10 anos entre os momentos de coletas de dados, período em que houve enorme expansão dos serviços básicos de saúde na região, principal responsável pela oferta deste tipo de cuidado.

Um dado que talvez possa servir de baseline para não realização deste exame é aquele provido pela PNAD 2008, que encontrou prevalência de não realização de citopatológico de $13 \%$ entre mulheres com idade entre 25 e 64 anos $^{25}$. A este valor devem ser acrescidas aquelas com exame em atraso podendo, desta forma, chegar mais próximo da prevalência encontrada neste estudo. $\mathrm{O}$ fato é que, apesar de estarem na idade de realização deste exame, com muitas delas inclusive em atraso, e de terem visitado o médico pelo menos uma vez durante o pré-natal, duas em cada 10 gestantes residentes no município de Rio Grande não foram submetidas a este exame.

Puérperas com até oito anos de escolaridade mostraram prevalência cerca de duas vezes maior de não realizar $\mathrm{CP}$ em relação àquelas com 12 anos ou mais de escolaridade. A escolaridade é um dos mais importantes determinantes das condições de saúde materno-infantil. Quanto maior a escolaridade da mulher, melhor os cuidados com a sua saúde, maior a busca por serviços de diagnóstico precoce, e mais precocemente se procura pelo cuidado médico quando de alguma necessidade ${ }^{26,27}$.

Tomando por base vários outros estudos que buscaram medir o efeito independente da escolaridade sobre a não realização de citopatológico de colo uterino, todos mostraram, mesmo após ajuste para diversos confundidores, medidas de efeito expressivas revelando a importância desta variável em relação a este desfecho ${ }^{5,9-12,28-30}$. Nestes estudos já mencionados, mulheres com até oito anos de escolaridade mostraram probabilidade pelo menos $50 \%$ maior de não realizar $\mathrm{CP}$ em relação às demais.

Dentre aquelas que relataram ocorrência prévia de aborto (induzido ou espontâneo), a prevalência de não se submeterem a este exame foi cerca de 1,4 vezes maior quando comparada às demais. Ter tido dois ou mais abortos também se mostrou significativamente associado a não realização de $\mathrm{CP}$ entre aquelas mulheres atendidas em uma área coberta pela Estratégia Saúde da Família ${ }^{29}$.

Puérperas que consumiram álcool durante a gravidez apresentaram prevalência de aproximadamente 1,4 vezes maior à não realização de Papanicolaou em relação a não usuárias de álcool durante a gravidez. Não se encontrou estudo publicado em que o álcool tenha se mantido, após ajuste, como fator de risco a não realização deste exame. Há que considerar que indivíduos que fazem uso rotineiro do álcool têm menor autocuidado, e este exame pode ser incluído neste grupo já que se trata da busca precoce por um possível problema de saúde ${ }^{31}$. Isto, no entanto, carece de consistência, daí a necessidade de outros estudos investigando esta variável.

Realizar menos de seis consultas de pré-natal aumentou a prevalência em 1,35 vezes de não realizar $\mathrm{CP}$ em relação àquelas que completaram sete ou mais consultas de pré-natal. Como mencionado anteriormente, em um sistema de saúde em que os programas de rastreamento são predominantemente oportunísticos, a procura por cuidado está diretamente ligado ao aumento da cobertura. Nesse sentido, fazer mais consultas aumenta a chance de realizar CP. Isto ocorreu em Juiz de Fora, MG, local em que a realização de 11 consultas no pré-natal mostrou-se protetor à realização 
de Papanicolaou ${ }^{23}$. Talvez não fosse necessário um numero tão elevado de consultas para evidenciar proteção.

Além do fato de $21,6 \%$ delas gestantes são terem realizado $\mathrm{CP}$, quando tinham de fazê-lo, o que chama a atenção neste estudo é que aproximadamente $90 \%$ delas terem completado pelo menos seis consultas de pré-natal e, mesmo assim, proporção expressiva não foi submetida a este exame. Isto sugere que a crescente e oportuna expansão dos serviços de atenção básica em saúde não se acompanhou do aproveitamento de situações em que se poderia aumentar a cobertura de serviços, dentre os quais a detecção precoce de câncer de colo uterino. Isto, por certo, contribui para a manutenção em níveis elevados da taxa de mortalidade por câncer de colo não somente no Brasil com em outros países de baixa e média rendas ${ }^{13,32}$.

Este desafio precisa ser enfrentado de forma mais contundente, organizada e resolutiva, ou se seguirá assistindo a ocorrência de mortes facilmente preveníveis país afora.

\section{Colaborações}

RJ Terlan e JA Cesar participaram igualmente de todas as etapas de elaboração do artigo. 


\section{Referências}

1. Instituto Nacional do Câncer (INCA). Estimativa 2014. Incidência de câncer no Brasil. Rio de Janeiro: INCA; 2014.

2. Brasil. Ministério da Saúde (MS). Controle dos Cânceres do colo do útero e da mama. Brasília: MS; 2013.

3. Instituto Nacional do Câncer (INCA). Inquérito domiciliar sobre comportamentos de risco e morbidade referida de doenças e agravos não transmissíveis: Brasil, 15 capitais e Distrito Federal, 2002-2003. Rio de Janeiro: INCA; 2004.

4. Quadros CAT, Victora CG, Costa JSD. Coverage and focus of a cervical cancer prevention program in Southern Brazil. Rev Panam Salud Publica 2004; 16(4):223232.

5. Cesar JA, Santos GB, Sutil AT, Cunha CF, Dumith SC. Citopatológico de colo uterino entre gestantes no Sul do Brasil: um estudo transversal de base populacional. Rev Bras Ginecol Obstet 2012; 34(11):518-523.

6. Martins LFL, Thuler LCS, Valente JG. Cobertura do exame de Papanicolaou no Brasil e seus fatores determinantes: uma revisão sistemática da literatura. Rev Bras Ginecol Obstet 2005; 27(8):485-492.

7. Correa MS, Silveira DS, Siqueira FV, Facchini LA, Piccini RX, Thumé E, Tomasi E. Cobertura e adequação do exame citopatológico de colo uterino em estados das regiões Sul e Nordeste do Brasil. Cad Saude Publica 2012; 28(12):2257-2266.

8. Albuquerque KM, Frias PG, Andrade CLT, Aquino EML, Menezes G, Szwarcwald CL. Cobertura do teste de Papanicolaou e fatores associados à não-realização: um olhar sobre o Programa de Prevenção do Câncer do Colo do Útero em Pernambuco, Brasil. Cad Saude Publica 2009; 25(Supl. 2):S301-S309.

9. Hackenhaar AA, Cesar JA, Domingues MR. Exame citopatológico de colo uterino em mulheres com idade entre 20 e 59 anos em Pelotas, RS: prevalência, foco e fatores associados à sua não realização. Rev Bras Epidemiol 2006; 9(1):103-111.

10. Cesar JA, Horta BL, Gomes G, Houlthausen RS, Willrich RM, Kaercher A, Iastrenski FM. Fatores associados à não realização de exame citopatológico de colo uterino no extremo Sul do Brasil. Cad Saude Publica 2003; 19(5):1365-1372.

11. Gasperin SI, Boing AF, Kupek E. Cobertura e fatores associados à realização do exame de detecção do câncer de colo de útero em área urbana no sul do Brasil: estudo de base populacional. Cad Saude Publica 2011; 27(7):1312-1322

12. Oliveira MMHN, Silva AAM, Brito LMO, Coimbra LC. Cobertura e fatores associados a não realização do exame preventivo de Papanicolaou em São Luís, Maranhão. Rev Bras Epidemiol 2006; 9(3):325-334

13. Instituto Nacional do Câncer (INCA). Atlas de Mortalidade por Câncer. [periódico na internet]. 2014. [acessado 2015 Jul 15]. Disponível em https://mortalidade. inca.gov.br/MortalidadeWeb

14. Instituto Nacional de Câncer José Alencar Gomes da Silva (INCA). Avaliação de indicadores das ações de detecção precoce dos cânceres do colo do útero e de mama - Brasil e regiões, 2013. Rio de Janeiro: INCA; 2015.
15. Anttila A, von Karsa L, Aasmaa A, Fender M, Patnick J, Rebolj M, Nicula F, Vass L, Valerianova Z, Voti L, Sauvaget C, Ronco G. Cervical cancer screening policies and coverage in Europe. Eur J Cancer 2009; 45(15):2649-2645.

16. Victora CG, Aquino EM, Carmo Leal M, Monteiro CA, Barros FC, Szwarcwald CL. Maternal and child health in Brazil: progress and challenges. Lancet 2011; 377(9780):1863-1876

17. Dean AG, Dean JA, Coulombier D, Brendel KA, Smith DC, Burton AH. Epi-Info, Version 6: A Word Processing, Database, and Statistics Program for Epidemiology on Microcomputers. Atlanta: Centers of Disease Control and Prevention; 2002.

18. Stata Corp. Stata statistical software: release 11.2. College Station: Stata Corporation; 2011.

19. Barros AJ, Hirakata VN. Alternatives for logistic regression in cross-sectional studies: an empirical comparison of models that directly estimate the prevalence ratio. BMC Med Res Methodol 2003; 3:21

20. Victora CG, Huttly SR, Fuchs SC, Olinto MT. The role of conceptual frameworks in epidemiological analysis: a hierarchical approach. Int J Epidemiol 1997; 26(1):224-227.

21. Kirkwood BR, Sterne JAC. Essential Medical Statistics Oxford: Blackwell; 2003.

22. Caplan LS, McQueen DV, Qualters JR, Leff M, Garrett C, Calonge N. Validity of women's self-reports of cancer screening test utilization in a managed care population. Cancer Epidemiol Biomarkers Prev 2003; $12(11$ Pt 1):1182-1187.

23. Ribeiro L, Bastos RR, Ribeiro LC, Vieira MT, Leite IC, Teixeira MT. Não adesão às diretrizes para rastreamento do câncer do colo do útero entre mulheres que frequentaram o pré-natal. Rev Bras Ginecol Obstet 2013; 35(7):323-330.

24. Gonçalves CV, Duarte G, Dias da Costa JS, Quintana SM, Marcolin AC. Perdas de oportunidades na prevenção do câncer de colo uterino durante o pré-natal. Cien Saude Colet 2011; 16(5):2501-2510.

25. Martínez-Mesa J, Werutsky G, Campani RB, Wehrmeister FC, Barrios $\mathrm{CH}$. Inequalities in Pap smear screening for cervical cancer in Brazil. Prev Med 2013; 57(4):366-371.

26. Travassos C, Viacava F, Pinheiro R, Brito A. Utilization of health care services in Brazil: gender, family characteristics, and social status. Rev Panam Salud Publica 2002; 11(5-6):365-373

27. Haidar FH, Oliveira UF, Nascimento LFC. Maternal educational level: correlation with obstetric indicators. Cad Saude Publica 2001; 17(4):1025-1029.

28. Amorim VMSL, Barros MBA, César CLG, Carandina $\mathrm{L}$, Goldbaum M. Fatores associados à não realização do exame de Papanicolaou: um estudo de base populacional no Município de Campinas, São Paulo, Brasil. Cad Saude Publica 2006; 22(11):2329-3829.

29. Augusto EF, Rosa ML, Cavalcanti SM, Oliveira LH. Barriers to cervical cancer screening in women attending the Family Medical Program in Niterói, Rio de Janeiro. Arch Gynecol Obstet 2013; 287(1):53-58. 
30. Borges MFSO, Dotto LMG, Koifman RJ, Cunha MA, Muniz PT. Prevalência do exame preventivo de câncer do colo do útero em Rio Branco, Acre, Brasil, e fatores associados à não-realização do exame. Cad Saude Publica 2012; 28(6):1156-1166.

31. Machado IE, Lana FCFL, Felisbino-Mendes MS, Malta DC. Factors associated with alcohol intake and alcohol abuse among women in Belo Horizonte, Minas Gerais State, Brazil. Cad Saude Publica 2013; 29(7):1449-1459.

32. Tsu V, Jeronimo J. Saving the World's Women from Cervical Cancer. N Engl J Med 2016; 374(26):2509-2510.

Artigo apresentado em 02/02/2016

Aprovado em 06/01/2017

Versão final apresentada em 08/01/2017 